\title{
The study of rolling stock wheels impact on rail switch frogs
}

\author{
Vadim Korolev ${ }^{1 *}$ \\ ${ }^{1}$ Russian University of Transport (MIIT), Chasovaya str. 22/2, Moscow, 125190, Russia
}

\begin{abstract}
Studies of stress distribution across cross sections by polarization-optical method made it possible to propose a relatively simple experimental method of determining contact forces. Experimental determination of contact forces on crossbars of switches is possible by means of sensors located in places of stress concentration (at core base). Analysis of oscillations during impact-pulse loading makes it possible to determine design parameters of the path. Typically, single circuits with four to five degrees of freedom produce quite satisfactory results and may be recommended for the calculated determination of contact forces on the crossbars of the switches.
\end{abstract}

\section{Introduction}

Increase of train speeds and increase of their axial loads lead to premature release of crossbars of switches due to contact-fatigue damages $[1,2]$.

Contact effects of wheels of rolling stock on crosses are determined by theoretical method using analog computers. This is because the existing methods of experimental determination of contact effects are very cumbersome due to the specificity of the switch frogs design $[3,4]$.

\section{Research technique}

Studies of stress distribution along cross sections by polarization-optical method made it possible to propose relatively simple experimental method of determining contact forces $[22,23]$. Figure 1 shows the pattern of isochromatic curves (lines of the same principal stress difference) in one of the cross-piece core sections under the influence of the carriage wheel. From the figure it can be seen that points 1 and 2 on the core cross-section contour are concentrators of stresses from contact action. Analysis of numerous versions of action of different wheels showed that the sum of contour stresses at these points is directly proportional to the value of vertical component of contact force and does not depend on the place of its application on rolling surface, as well as on horizontal transverse component of this force. Therefore, vertical components of contact forces were fixed. In order to avoid the effect of bending stresses, the sensors were adhered vertically to the core. Fixation was carried out in the core sections 12, 20, 30, 40 and $70 \mathrm{~mm}$. To obtain the sum of the

${ }^{*}$ Corresponding author: shishkinaira@inbox.ru 
voltages, the sensors glued at points 1 and 2 were connected to one arm of the measuring bridge. From each wheel of the pilot train in all sections there is a clear peak on the oscillogram.

Amplitude corresponds to value of contact force. Calibration of measuring channels was performed by rolling of carriages with known loads from wheel onto corresponding frog cross section.

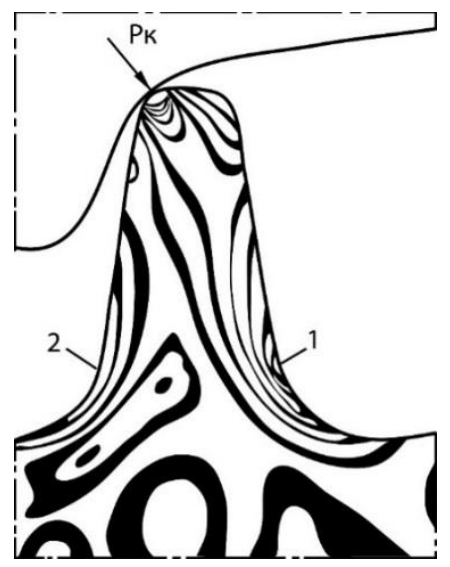

Fig. 1. Isochromic pattern in $20 \mathrm{~mm}$ core cross section of new switch frog from effect of worn wheel. Dark stripes - curves with similar difference in principal stresses

The average values of contact forces obtained by the described method in the section of 30 $\mathrm{mm}$ of the cross-bar of P65 grade 1/11 from the impact of 4-axle gondola cars with a load on the axle of 23 tons were about 20 tons at a speed of $70 \mathrm{~km} / \mathrm{h}$. The crosspiece on which the measurements were made had a core wear of $40 \mathrm{~mm}$ in section of $2.1 \mathrm{~mm}$. Roughness is a shallow cavity of semi-sinusoidal shape.

If the sensors are labelled correctly, there is no influence of extraneous factors; In such a case, the width of the peak corresponds to the length of the pad [5, 6, 7]. The statistical comparison of land lengths obtained by the described method is well consistent with the data obtained by the re-costing using in-kind wheel marks on the cross.

Unfortunately, the proposed method of experimental determination of contact forces on crosses does not allow to determine contact forces of influence on wing rails. In addition, it, like most experimental methods in general, requires a lot of money and time for implementation, especially in view of the large number of possible options for passing wheels on switch frogs $[8,18]$. Therefore, the theoretical method of determining contact forces, taking into account recommendations, should be considered the main method at present. One of the most important issues in determining contact forces by calculation method is selection of calculation scheme $[9,10,19,20]$. A flat mechanical system with 11 degrees of freedom and nonlinear connections between the elements was used as the base to investigate the impact of the design scheme on the results of contact forces (Figure 2). The main difference between it and the previous schemes is the introduction of additional connection between the elements modeling the cross-piece, which allows to more fully investigate the influence of the second wheel of the car trolley when rolling carriages along the crossings. The connections in the wheel-crossing contact and in the track elements were modeled as one-way, which allowed us to study the carriage-track system behavior during wheel separation and the subsequent process of shock-pulse interaction of the wheels with the crossing, which takes place in real conditions in the rolling zone $[11,12]$. As perturbing functions, the trajectories of rolling the wheels along the crossings, constructed using the diameters of real wheels and frogs, were used.

Rolling trajectories were formed by piecewise linear approximation using nonlinear 
blocks. The model provides for a separate entry of the rolling paths of each wheel, which allows us to study the vibrations of the "carriage-track" system in the presence of wheels with different shapes and sizes of wear in one cart, as well as with different positions of the cart wheels in the gutter of the frog. In calculating the derivative of the vertical roughness of the path $\eta$, inertial links were used instead of differentiators, which avoided an abrupt change in the value of $\eta$ in the places of fractures on the trajectories and at the same time increased the stability of the scheme as a whole.

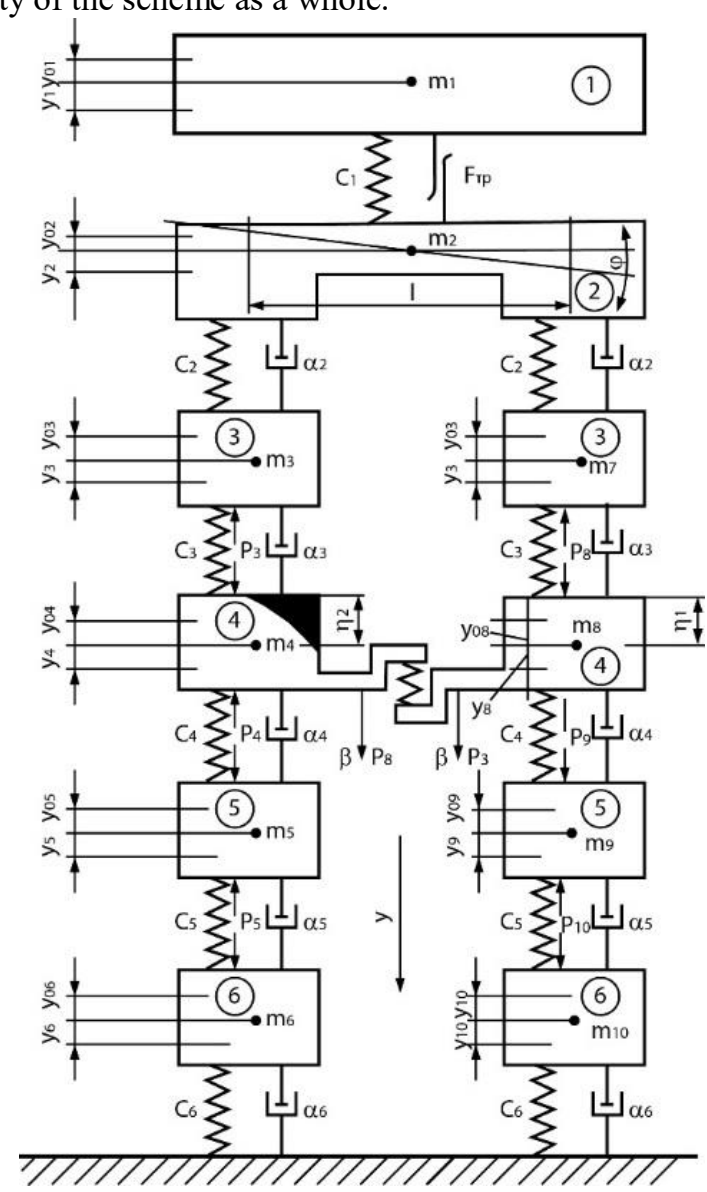

Fig. 2. Design scheme with 11 degrees of freedom: 1 - body; 2 - frame with axle box; 3 - part of wheel rim involved in interaction; 4 - frog; 5 - timber; 6 - part of timber base involved in interaction

The behavior of the calculated mechanical system is described by 11 second-order differential equations and six auxiliary equations. In this case, three types of design schemes were investigated: linear with the number of degrees of freedom up to six, not taking into account the second wheel of the cart; flat with the number of degrees of freedom up to 11 , taking into account the influence of the neighboring axis due to interaction only through the cart elements $(\beta=0)$, and flat with the number of degrees of freedom up to 11 , taking into account the influence of wheels through the elements of the cart and the path. The study was carried out in the speed range $60-120 \mathrm{~km} / \mathrm{h}$.

The most difficult issue in modeling the carriage-track system is the choice of design parameters $[13,14,21]$. The calculated parameters relating to the track in the area of rail switch frogs were determined experimentally. The stiffnesses of the links of the track elements were obtained according to the results of static tests conducted at the Department 
of Transport Construction of the Russian University of Transport. In this case, the stiffnesses of both bonds in the ballast ( $c_{5}$ and $c_{6}$ in Fig. 2 ) are assumed to be the same.

To determine the masses of the elements of the path and the coefficients of viscous friction, a method was developed based on the analysis of path elements vibrations. The dynamic equilibrium of the mass modeling a frog in the design scheme (Fig. 3, a) is determined by the equation:

$$
M_{w c} \ddot{y}=F_{\mathrm{\kappa}}-\sum_{i=1}^{n} F_{j}^{0}
$$

wherein $M_{w c}$ - frog mass; $\ddot{y}-$ frog acceleration; $F_{\kappa}$ - contact force; $\sum_{i=1}^{n} F_{j}^{0}-$ total reaction of frog base.
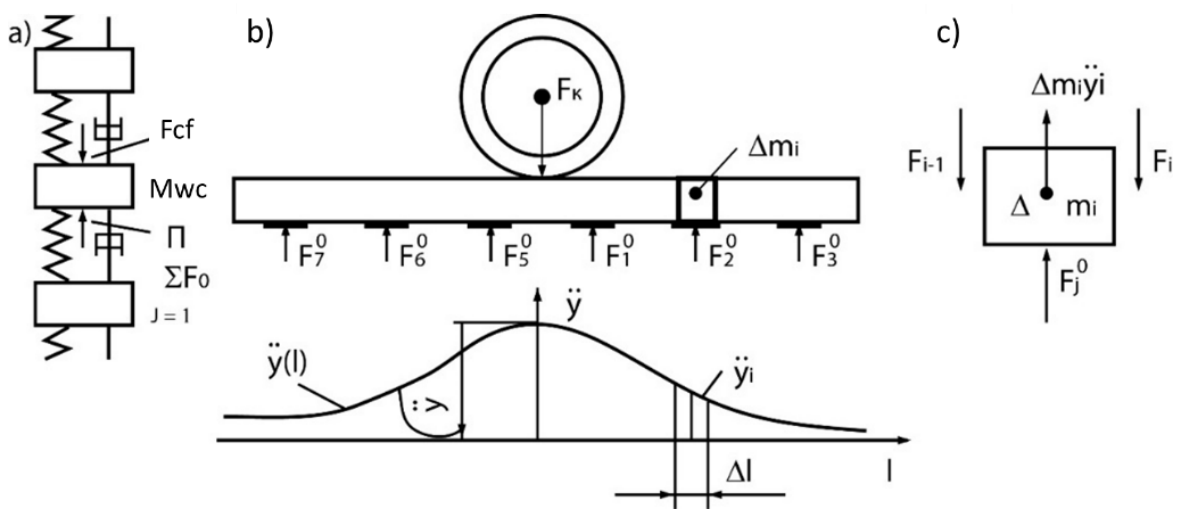

Fig. 3. Schemes for experimental method for determining design parameters of frog unit: a) theoretical wheel-frog system; b) forces acting on crossing and diagram of accelerations; c) system of forces acting on $\mathrm{i}$-th element

If we consider a crossing as a beam consisting of a large number of elements lying on $n$ elastic supports (Fig. 3b), then the equation of dynamic equilibrium of the i-th element (Fig. 3c) will have the form:

$$
\Delta m_{i} \ddot{y}_{l}=-F_{j}^{0}+F_{i-1}+F_{i}
$$

wherein $\Delta m_{i}-$ mass of $i$-th element; $\ddot{y}_{l}-$ acceleration of i-th element; $F_{j}^{0}$ - base reaction; $F_{i}, F_{i-1}$ - internal forces connecting the elements together. Given that the internal forces acting on neighboring elements are equal in magnitude and directed in opposite directions, we have:

$$
\sum_{i=1}^{l} \Delta m_{i} \ddot{y}_{l}=F_{k}-\sum_{j=1}^{n} F_{j}^{0}
$$

The right-hand sides of equations (1) and (3) are equal. Wherefrom we get:

$$
M_{w c}=\frac{\sum_{i=1}^{l} F_{j}^{0}}{\ddot{y}}
$$

The mass of the i-th element can be represented as follows:

wherein $\mu$ - linear mass of crossing.

$$
\Delta m_{i}=\mu \Delta l_{i}
$$

Substituting the value $\Delta m$ in equation (4) and going over to the limit, as well as assuming conditionally that $\mu$ is a constant value, we finally obtain:

$$
M_{w c}=\mu \frac{\int_{-\infty}^{+\infty} \ddot{y}(l) d l}{\ddot{y}}
$$

The integral in the numerator of equation (6) is the area of the diagram of the crossing accelerations, and the value of $\ddot{y}$ is the acceleration in the section directly under the wheel taken at the same moment in time. As noted above, on the frogs in the rolling zone there is an impact interaction process, therefore, vibration measuring equipment was used to experimentally plot acceleration diagrams. Plotting vibration accelerations directly under a 
moving train load is difficult due to the imposition of vibrations caused by different wheels, therefore, to create a shock pulse, the wheel of a 4-axle gondola was dumped from a wedge $20 \mathrm{~mm}$ high onto a frog at a low speed (less than $5 \mathrm{~km} / \mathrm{h}$ ). The reduced masses of the beams and ballast were also determined $[15,16,17]$. For ballast, volumetric weight and volume integral - volumetric acceleration diagram - were substituted into equation (6).

The coefficients of viscous friction $\alpha$ were determined by the rate of damping the vibrations, while the ratio of the amplitudes of the vibration displacements was replaced by the corresponding ratio of the amplitudes of the vibration accelerations.

Coefficient $\beta$ takes into account the influence of the neighboring axis; it shows how much pressure is transferred to the base of the cross under the second wheel from the effects of the first wheel. This coefficient was determined by the calculation method using tables of influencing lines for the frog as for a beam with $n$ elastic supports.

\section{Results and discussion}

The numerical values of the calculated parameters were obtained as follows: mass $\left(\mathrm{kgf} \cdot \mathrm{s}^{2} /\right.$ cm) $m_{1}=19.80 ; m_{2}=0.735 ; \mathrm{m}_{3}=\mathrm{m}_{7}=0.173 ; \mathrm{m}_{4}=\mathrm{m}_{8}=0.420 ; m_{5}=m_{9}=0.400 ; m_{6}=m_{10}$ $=2.500$; friction force $\mathrm{F}_{\mathrm{tr}}=1500 \mathrm{kgf}$; coefficient of viscous friction $(\mathrm{kgf} \cdot \mathrm{s} / \mathrm{cm}) \mathrm{a}_{2}=60$; $\mathrm{a}_{3}=100 ; \mathrm{a}_{4}=83 ; \mathrm{a}_{5}=132 ; a_{6}=308 ;$ bond stiffness $(\mathrm{kgf} / \mathrm{cm}) \mathrm{s}_{1}=0.2 \cdot 10^{5} ; \mathrm{c}_{2}=5.0 \cdot 10^{5} ;$ $\mathrm{c}_{3}=10.0 \cdot 10^{5} ; \mathrm{c}_{4}=1.59 \cdot 10^{5} ; \mathrm{c}_{5}=2.5 \cdot 10^{5} ; \mathrm{c}_{3}=2.5 \cdot 10^{5} ;$ moment of inertia $I=3240$ $\mathrm{kgf} \cdot \mathrm{cm} \cdot \mathrm{s}^{2}$; distance $l=185 \mathrm{~cm}$; coefficient $\beta=0.20$.

An analysis of the calculated contact forces of pressure on the frog showed that taking into account the influence of the adjacent axis only through the cart elements (second-type circuits) leads to an increase in the values of the dynamic additions of contact forces by an average of 1.26 times as compared to similar values obtained on the same rolling paths with using linear circuits. Taking into account the additional influence of the neighboring axis through the elements of the path leads to a decrease in the addition of contact forces by an average of 1.3 to 1.4 times. Thus, taking into account the influence on the interaction forces of the neighboring axis of the trolley through the elements of the path on average eliminates the difference between the contact forces obtained with schemes that take into account and do not take into account the neighboring second wheel. However, it should be noted that in some specific cases such a leveling may not occur, especially in the case when the wheels of one trolley are rolled along the crossing, occupying different positions in its trenches.

\section{Conclusions}

1. Experimental defining of the contact forces on the rail switch frogs is possible with the help of sensors located in places of stress concentration (at the base of the core).

2. The studying of oscillations in the process of shock-pulse loading allows us to determine the calculated path parameters.

3. As a rule, single circuits with four to five degrees of freedom give quite satisfactory results and can be recommended for the calculation of contact forces on the rail switch frogs.

\section{References}

1. Loktev, V. Korolev et al. Procedia Engineering, 189, 133-137 (2017) DOI: 10.1016/j.proeng.2017.05.022 
2. B. Glusberg, V. Korolev et al. MATEC Web of Conferences, 239, 01054 (2018) DOI: 10.1051/matecconf/201823901054

3. A. Loktev, V. Korolev, I. Shishkina. IOP Conf. Series: Materials Science and Engineering, 463, 032019 (2018) DOI: 10.1088/1757-899X/463/3/032019

4. A. Loktev, V. Korolev et al. IOP Conf. Series: Materials Science and Engineering, 463, 032018 (2018) DOI: 10.1088/1757-899X/463/3/032018

5. B. Glusberg, A. Savin, A. Loktev et al. Advances in Intelligent Systems and Computing, 982, 556-570 (2020) DOI: 10.1007/978-3-030-19756-8_53

6. V. Korolev. TransSiberia 2019: VIII International Scientific Siberian Transport Forum, 621-638 DOI: 10.1007/978-3-030-37916-2_60

7. B. Glusberg, A. Savin, A Loktev et al. Advances in Intelligent Systems and Computing, 982, 571-587 (2020) DOI: 10.1007/978-3-030-19756-8_54

8. I. Shishkina. TransSiberia 2019: VIII International Scientific Siberian Transport Forum, 834-844, DOI: 10.1007/978-3-030-37916-2_82

9. A. Loktev, V. Korolev, I. Shishkina et al. Advances in Intelligent Systems and Computing, 982, 325-336 (2020) DOI: 10.1007/978-3-030-19756-8_30

10. B. Gluzberg, V. Korolev, A. Loktev et al. E3S Web of Conferences, 138, 01017 (2019) DOI: $10.1051 / \mathrm{e} 3$ sconf $/ 201913801017$

11. A. Loktev, V. Korolev et al. Vestnik of the Railway Research Institute, 77(2), 77-83 (2018) DOI: 10.21780/2223-9731-2018-77-2-77-83

12. A. Loktev, V. Korolev et al. Vestnik of the Railway Research Institute, 77(6), 331-336 (2018) DOI: 10.21780/2223-9731-2018-77-6-331-336

13. V. Korolev. TransSiberia 2019: VIII International Scientific Siberian Transport Forum, 175-187, DOI: 10.1007/978-3-030-37919-3_17

14. A.V. Savin, V.V. Korolev, I.V. Shishkina. IOP Conf. Series: Materials Science and Engineering, 687, 022035 (2019) DOI: 10.1088/1757-899X/687/2/022035

15. A. Savin, A. Kogan et al. International Journal of Innovative Technology and Exploring Engineering, 8(7), 2325-2328 (2019)

16. A.A. Loktev, V.V. Korolev, E.A. Gridasova. IOP Conf. Series: Materials Science and Engineering, 687, 022036 (2019) DOI: 10.1088/1757-899X/687/2/022036

17. V. Korolev, A. Loktev et al. IOP Conference Series: Earth and Environmental Science, 403, DOI: 10.1088/1755-1315/403/1/012194

18. E. Gridasova, P. Nikiforov et al. TransSiberia 2019: VIII International Scientific Siberian Transport Forum, 559-569, DOI: 10.1007/978-3-030-37916-2_54

19. A.Savin, O. Suslov et al. TransSiberia 2019: VIII International Scientific Siberian Transport Forum, 648-654, DOI: 10.1007/978-3-030-37916-2_62

20. A. Savin, V. Korolev et al. TransSiberia 2019: VIII International Scientific Siberian Transport Forum, 797-808, DOI: 10.1007/978-3-030-37916-2_78

21. A. Loktev, V. Korolev, I. Shishkina et al. TransSiberia 2019: VIII International Scientific Siberian Transport Forum, 2, 1116 (2020) DOI: 10.1007/978-3-030-37919-3

22. B. Glusberg, A. Loktev et al. International Scientific Conference Energy Management of Municipal Facilities and Sustainable Energy Technologies EMMFT 2018, 337-345 (2020) DOI: 10.1007/978-3-030-19756-8_31

23. A. Lyudagovsky, A. Loktev, V. Korolev et al. E3S Web of Conferences, 110, 01017 (2019) DOI: 10.1051/e3sconf/201911001017 